\title{
Calculation of plates in a geometrically nonlinear setting with the use of generalized equations of finite difference method
}

\author{
Natalia Uvarova ${ }^{1, *}$, and Radek Gabbasov ${ }^{1}$ \\ ${ }^{1}$ Moscow State University of Civil Engineering, Yaroslavskoe shosse, 26, 129337, Moscow, Russia
}

\begin{abstract}
The article proposes a numerical method and an algorithm for analysis rectangular flexible plates in a geometrically nonlinear formulation. The generalized equations of the method of finite differences (MD) are used to solve the problem within the integrable region taking into account the discontinuities of the desired function, its first derivative and the right part of the original differential equation. Solving differential equations of the problem, composed with respect to the desired functions of deflection and stress are reduced to the 4th differential equations of the second order, which are solved numerically. As an example, a square plate loaded with a uniformly distributed load is considered. The results of the calculation with a minimum number of partitions are compared with the known analytical solution of A. S. Volmir [1] and indicate the possibility of using the numerical method for solving problems in a nonlinear formulation.
\end{abstract}

\section{Introduction}

When designing large-span frames of industrial buildings and structures, special attention should be paid to the strength and rigidity of the main load-bearing structures.

Quite a large number of works are devoted to the calculation of thin plates in geometrically nonlinear formulation. B [1]-[3] variation methods of Galerkin, RitzTymoshenko are used, in [4] an approximate method of calculation is developed, in which a

"Corresponding author: nbuvarova@,yandex.ru 
correction to a linear solution is found when solving a nonlinear problem, in [5] a nonlinear incremental structural mechanics is used. The most common and universal numerical method for calculating such structures (including nonlinear calculations) is the extrafamous finite element method (FEM) [6] - [12].

\section{Calculation}

The calculation of the overlaps of these structures taking into account large deflections, that is, in a geometrically nonlinear formulation can be performed using the technique proposed below.

The purpose of the work is to develop an algorithm for the numerical method of solving the nonlinear problem of calculation and illustrate it by example with a minimum number of partitions of the region or on a rare grid using generalized equations of the finite difference method.

A thin isotropic square plate with elastic deflections comparable to the thickness is considered, that is, a geometrically nonlinear problem is formulated.

The calculation of flexible plates operating on a transverse load [1] is reduced to the solution of two nonlinear partial differential equations of the fourth order (a system of nonlinear differential equations of the theory of flexible plates) formulated with respect to the deflections $\mathrm{W}$ and the stress function $\Phi$ :

$$
\begin{gathered}
\frac{D}{H} \nabla^{2} \nabla^{2} W=\frac{\partial^{2} \Phi}{\partial y^{2}} \frac{\partial^{2} W}{\partial x^{2}}+\frac{\partial^{2} \Phi}{\partial x^{2}} \frac{\partial^{2} W}{\partial y^{2}}-2 \frac{\partial^{2} \Phi}{\partial x \partial y} \frac{\partial^{2} W}{\partial x \partial y}+\frac{q}{H} \\
\nabla^{2} \nabla^{2} \phi=E\left[\left(\frac{\partial^{2} W}{\partial x \partial y}\right)^{2}-\frac{\partial^{2} W}{\partial x^{2}} \frac{\partial^{2} W}{\partial y^{2}}\right]
\end{gathered}
$$

Here $\mathrm{W}$ is the deflection, $\Phi-$ is the stress function, $\mathrm{H}$ is the plate thickness, $\mathrm{D}$ is the cylindrical rigidity, $\mathrm{q}$ is the intensity of the distributed transverse load, $\mathrm{E}$ is the modulus of elasticity.

Introduce the dimensionless parameters:

$$
\xi=\frac{x}{a}, \eta=\frac{y}{a}-\text { dimensionless coordinates; } w=\frac{W}{a} ; f=\frac{\Phi}{\mathrm{E} \cdot a^{2}}
$$

where $\mathrm{a}$ is the length of the side of the plate.

After a series of transformations (1) and (2) we obtain: 


$$
\begin{gathered}
\left(\frac{\partial^{2}}{\partial \xi^{2}}+\frac{\partial^{2}}{\partial \eta^{2}}\right)\left(\frac{\partial^{2} w}{\partial \xi^{2}}+\frac{\partial^{2} w}{\partial \eta^{2}}\right)=12\left(1-\mu^{2}\right) \frac{a^{2}}{H^{2}}\left(\frac{\partial^{2} f}{\partial \eta^{2}} \frac{\partial^{2} w}{\partial \xi^{2}}+\frac{\partial^{2} f}{\partial \xi^{2}} \frac{\partial^{2} w}{\partial \eta^{2}}-2 \frac{\partial^{2} f}{\partial \xi \partial \eta} \frac{\partial^{2} w}{\partial \xi \partial \eta}\right)+\frac{a^{3}}{D} q \\
-\left(\frac{\partial^{2}}{\partial \xi^{2}}+\frac{\partial^{2}}{\partial \eta^{2}}\right)\left(\frac{\partial^{2} f}{\partial \xi^{2}}+\frac{\partial^{2} f}{\partial \eta^{2}}\right)=\left(\frac{\partial^{2} w}{\partial \xi^{2}}+\frac{\partial^{2} w}{\partial \eta^{2}}\right)-\left(\frac{\partial^{2} w}{\partial \xi \partial \eta}\right)^{2} \\
\text { Let us call } \frac{\partial^{2} w}{\partial \xi^{2}}+\frac{\partial^{2} w}{\partial \eta^{2}}=-m
\end{gathered}
$$

And a right part (4) through $g$ :

$$
g=\frac{a^{3}}{D} q+12\left(1-\mu^{2}\right) \frac{a^{2}}{h^{2}}\left(\frac{\partial^{2} f}{\partial \eta^{2}} \frac{\partial^{2} w}{\partial \xi^{2}}+\frac{\partial^{2} f}{\partial \xi^{2}} \frac{\partial^{2} w}{\partial \eta^{2}}-2 \frac{\partial^{2} f}{\partial \xi \partial \eta} \frac{\partial^{2} w}{\partial \xi \partial \eta}\right)
$$

Then (4) takes the form:

$$
\frac{\partial^{2} m}{\partial \xi^{2}}+\frac{\partial^{2} m}{\partial \eta^{2}}=-g
$$

We will do the same with equation (6).

$$
\text { Denote } \frac{\partial^{2} f}{\partial \xi^{2}}+\frac{\partial^{2} f}{\partial \eta^{2}}=s
$$

a right part (6) through $l$ :

$$
l=\frac{\partial^{2} w}{\partial \xi^{2}} \frac{\partial^{2} w}{\partial \eta^{2}}-\left(\frac{\partial^{2} w}{\partial \xi \partial \eta}\right)^{2}
$$

Then (6):

$$
\frac{\partial^{2} s}{\partial \xi^{2}}+\frac{\partial^{2} s}{\partial \eta^{2}}=l
$$

Thus, two differential equations of the fourth order, composed with respect to the desired deflection and stress functions, are reduced to the 4th differential equations of the second order (6), (8), (9), (11). 
Using the generalized equations of the FMD, we write the difference approximation of one of these equations, for example (8), for the General case in the presence of gaps of the desired function, its first derivative and the right side of the differential equation (3.1.24) [13]. We write this equation by multiplying all the values by $h^{2}$ and denoting

$$
\begin{gathered}
m=m h^{2} \\
m_{i-1, j}+m_{i, j-1}-4 m_{i, j}+m_{i, j+1}+m_{i+1, j}+\frac{h}{2}\left({ }^{I-I I} \Delta m_{i j}{ }^{\prime}+{ }^{I I-I V} \Delta m_{i j}+{ }^{I-I I I} \Delta m_{i j} \eta+{ }^{I I-I V} \Delta m_{i j}^{\eta}\right)= \\
=-\frac{h^{2}}{4}\left({ }^{I} p_{i j}+{ }^{I I} p_{i j}+{ }^{I I I} p_{i j}+{ }^{I V} p_{i j}\right)
\end{gathered}
$$

The fragment of the grid on which the solution is built is shown in figure 1. Derivatives included in the expression (7) and (10), determined by the known formulas FMD:

$$
\begin{aligned}
& \frac{\partial^{2} w}{\partial \xi^{2}}=\frac{1}{h^{2}}\left(w_{i-1, j}-2 w_{i, j}+w_{i+1, j}\right) \\
& \frac{\partial^{2} w}{\partial \xi \partial \eta}=\frac{1}{4 h^{2}}\left(-w_{i-1, j}+2 w_{i, j}+w_{i+1, j}\right)
\end{aligned}
$$

We present the algorithm of the solution.

Having defined $g=\frac{a^{3}}{D} q$ from (7) and solving equations (8) we define m; then from equations (6) we find w; having calculated $l$ by (10) we solve equations (9) and find $s$; then by (9) we define f and by (7)we calculate $g$ again; after that the process repeats.

When moving to a new cycle, we accept

$$
g=\frac{1}{2}\left(g_{n-1}+g_{n}\right)
$$

where $g_{n}$ and $g_{n-1}$ are the last and penultimate values of $g$ respectively.

At the found values of the deflection $\mathrm{w}$ and the stress function $\mathrm{f}$ it is possible to construct all the corresponding solutions by [1]. 
As an example, consider a hinged flexible square plate with movable edges loaded with a uniformly distributed load (figure 2). The plate is symmetric.

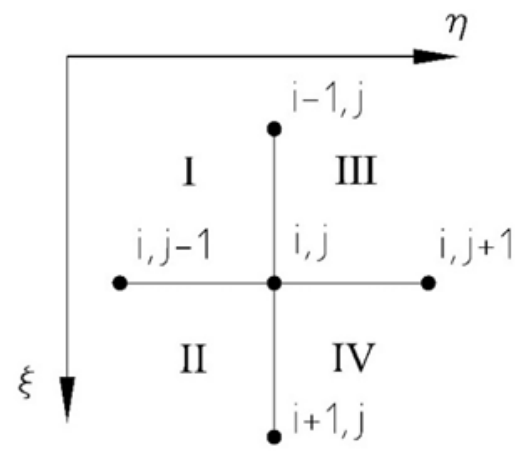

Fig. 1. A fragment of the mesh
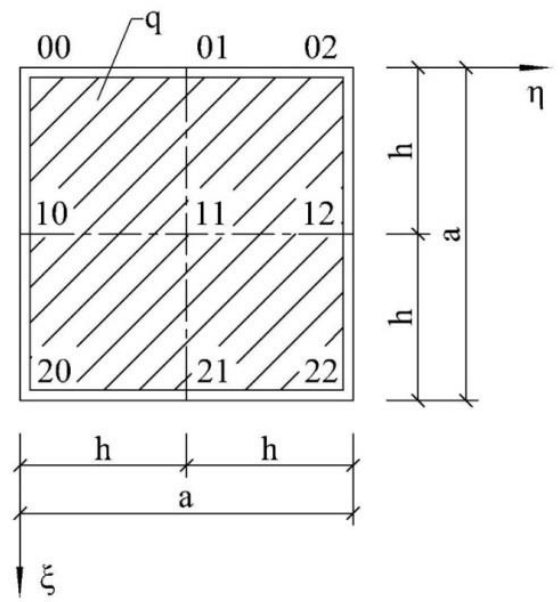

Fig. 2. Hinged plate under uniformly distributed load

Plate thickness $\mathrm{H}=0,1 \mathrm{~cm}$, side $\mathrm{a}=10 \mathrm{~cm}$, load $\mathrm{q}=0,5 \mathrm{~kg} / \mathrm{cm} 2, \mathrm{E}=0,75 \cdot 106 \mathrm{~kg} / \mathrm{cm} 2$ and $\mu=0,316$. Boundary conditions on the sides $\mathrm{x}=0$ and $\mathrm{x}=\mathrm{a}$ :

$$
W=0, M_{x}=0, \frac{\partial^{2} \Phi}{\partial y^{2}}=0, \frac{\partial^{2} \Phi}{\partial x \partial y}=0
$$

On the sides $\mathrm{y}=0$ and $\mathrm{y}=\mathrm{a}$ we get the same boundary conditions by changing $\mathrm{x}$ to $\mathrm{y}$. 
Consider a planar grid with a minimum number of partitions in steps $\mathrm{h}=0.5$. Figure 2 . Having determined the derivatives in the right part (7) of the FMD and taking into account the boundary conditions, we obtain:

$$
g_{11}=\frac{a^{3} q}{D}+96\left(1-\mu^{2}\right) \frac{a^{2}}{H^{2}} \frac{1}{h^{4}} f_{11^{w}} w_{11}
$$

Writing the difference approximation (8) to (12) and, given the boundary conditions, we obtain:

$$
m_{11}=\frac{h^{2}}{4} g_{11}
$$

Writing the difference approximation (6) to (12) and, given the boundary conditions, we obtain:

$$
w_{11}=\frac{h^{2}}{4} m_{11}
$$

Having determined the derivatives in the right part (10) according to the FMD, we obtain:

$$
l_{11}=\frac{4}{h^{4}} w_{11}^{2}
$$

Writing the difference approximation (11) to (12), replacing $\mathrm{m}$ and $\mathrm{p}$ by $\mathrm{s}$ and $l$, given the boundary conditions, we obtain:

$$
S_{11}=-\frac{h^{2}}{4} l_{11}
$$

Writing the difference approximation (9) and taking into account the boundary conditions, we obtain:

$$
f_{11}=\frac{h^{2}}{4} S_{11}
$$

Taking into account (17) and (18) from (19) we obtain:

$$
f_{11}=-\frac{1}{4} w_{11}^{2}
$$


Taking into account (14), (15) and (20) from (16) we obtain:

$$
\begin{gathered}
13498,88 w_{11}{ }^{3}+w_{11}-0,028=0 \\
w_{11}=0,0109 \\
w_{11}=w_{11} \cdot a=0,011 \cdot 10=0,11(\mathrm{~cm})
\end{gathered}
$$

It should be said that the solution of simply supported plates the values of $\mathrm{m}$ and $\mathrm{w}$ are determined without the involvement of the relations (18) and (19).

Let's compare the result with the solution of this problem in [1].

For a square plate with movable edges from equation (2.118) [1] we determine $\zeta=\frac{W}{H}$

$$
\begin{gathered}
7,5 \zeta^{3}+22 \zeta=q^{*} \\
q^{*}=\frac{q}{E}\left(\frac{a}{H}\right)^{4}\left(1-\mu^{2}\right)=\frac{0,5}{0,75 \cdot 10^{6}}\left(\frac{10}{0,1}\right)^{4}\left(1-0,316^{2}\right)=60
\end{gathered}
$$

Solving this equation for the above parameters, we find $\zeta=1,52$

$$
W=\zeta H=1,52 \cdot 0,1=0,152(\mathrm{~cm})
$$

The result is a rough approximation. However, for a two-dimensional geometrically nonlinear problem with a sparse grid with a step $h=0.5$, the solution can be considered satisfactory. Note that in [14] the solution of the one-dimensional axisymmetric problem for the calculation of a round plate at the step $h=0.5$ solution error was $9 \%$. The accuracy of the solution should be improved by reducing the grid spacing. The calculation algorithm is designed to use computers.

\section{Conclusions}

The example of calculation serves as an illustration of the algorithm and shows that the generalized equations of the MD give results that allow solving problems in a nonlinear formulation. The proposed technique can be used in computational practice. The developed calculation algorithm provides additional solutions to geometrically nonlinear problems along with other methods. 


\section{References}

1. A. S. Volmir, Moscow: State pub. house of tech and theoretic. lit., 420, (1956)

2. S. P. Timoshenko, and S. Voynovskiy-Kriger, IN, 635, (1966)

3. V. R. Kuliev, Thesis on VAK Perm, 138, (2000)

4. V. V. Rogalevich, S. A. Timashev ABU NIIPROEKT, Ekaterinburg, 52-56, (2012)

5. V. V. Petrov Ed. Infra-Engineering, 480, (2014)

6. A. B. Zolotov, P. A. Akimov., V. N., Sidorov, M. L. Mozgaleva, M: DIA, 336, (2009)

7. T. Belytscko, W. Liu, B. Moran, J Wiley \& Sons, 300, (2000)

8. J. Bonet, R. Wood, CUP, 248, (1997)

9. M.A. Crisfield, J Wiley\&Sons, 1, 362, (1997)

10. P. Kyther, D. Wie, BV, 445, (2004)

11. J.N. Reddy, OUP, 488, (2004)

12. A.N. Danilin, N.N. Zuev, D.V. Snegovsky, V.I. Shalashilin, 4, 26-31, (2000)

13. R. F. Gabbasov, A. R. Gabbasov., V. V. Filatov. Ph ASV, (2008)

14. R. F. Gabbasov, N. B. Uvarova, V. MGSU, 6(105), 631-635. (2017) 